\title{
Social and psychological responses to home treatment of haemophilia
}

\author{
JOSEPH M. KAUFERT \\ From the Department of Social and Preventive Medicine, University of Manitoba, Winnipeg
}

SUMMARY A pilot home treatment programme was set up in 1975 by the Departments of Haematology and Community Medicine, St. Thomas's Hospital, London, and the Haemophilia $\frac{\bar{\rho}}{\bar{D}}$ Centre, Churchill Hospital, Oxford. The purpose of the study was to assess the clinical, social, and $\stackrel{\odot}{\circ}$ economic effects of enabling haemophiliacs to treat themselves at home. The clinical and economic is aspects of the project have been discussed by Ingram et al. ${ }^{1}$ In this paper the immediate social and $\vec{\circ}$ psychological impact of the home treatment programme is described.

Self-treatment of haemophilia at home is becoming a widely practised alternative to the provision of outpatient treatment at special centres. The clinical advantages of home treatment are that it reduces the delay before therapy can begin ${ }^{1}$ and reduces arthropathy. ${ }^{2}$ Among its other advantages are the capacity to reduce time lost from school and work ${ }^{3}$ and to minimise the haemophiliac's dependence on outpatient clinics. ${ }^{4}$ The benefits to the patient also include freedom from the disruptions associated with having to make emergency trips to hospital and reduction in the treatment delays and pain which such crises entail. ${ }^{5}$

In the literature on the psychosocial difficulties that arise during patients' adaptation to haemophilia, there are three problems on which a home treatment programme was expected to have a major impact. These are:

(1) The limitation of the educational and occupational opportunities of haemophiliacs. ${ }^{6-9}$

(2) The pattern of dependence on family, on friends, and on clinical staff resulting from treatment requirements and disability associated with haemophilia. ${ }^{10}$

(3) The constraints on the development of functional skills ${ }^{11}$ and on the ability to take part in high-risk activities. ${ }^{12}$

The impact of self-therapy on educational and occupational opportunities may lie in the ability of the patient to treat himself at home, at work, or at school. The direct and indirect costs of treatment, including enforced absence from work or classroom, will be reduced. In the long run, the continuity of the individual's work or education may be improved. As the absence of this continuity has been a major $\stackrel{?}{?}$ barrier, home treatment should increase bot educational and vocational attainment. Howeveff $\dot{\omega}$ these potential benefits have to be weighed against $\overrightarrow{0}$ the costs of home treatment. The latter include the logistical, social, and psychological problems \& 응 performing venepunctures without direct clinic $\overrightarrow{\text { A }} \vec{A}$ supervision at home or at work. The small sample and $\mathbb{D}$ limited duration of the study restricted evaluation of the longer-term impact on occupational ang $\frac{\mathbb{1}}{3}$ educational opportunities. However, some. immediate advantages (such as a saving in time awa from school or work) and disadvantages (such as the $\vec{\varnothing}$ problem of obtaining help outside the clinical environment) were documented.

The second group of benefits which may result from home therapy are the increased control which the patient may achieve over his own treatment and the decrease in his dependence on the centre. These benefits have to be weighed against the social, psychological, and economic costs of the involvement of family and friends in the treatment. Dependency relations may simply be refocused away from professional helpers and towards members of the patient's informal network. In the study, measurements were made at four points in time of changes in patterns of perceived dependence. Finally, the study looked at the impact of home treatment on the patients' functional abilities and their willingness to take part in activities seen by them as involving greater risks.

\section{Methods}

DATA COLLECTION

To assess the effects of changing from outpatient clinics to home treatment, information on the 28 
severely affected patients, 12 from the treatment centre at Oxford and 16 from St. Thomas's Hospital, London, was gathered during three months of outpatient therapy and nine months of home therapy. Using a consistent research protocol and similar treatment procedures, home and outpatient treatment could be compared within each centre.* The schedule of interviews and the type of information gathered are summarised in the Figure. Questionnaires were administered by an interviewer at the beginning and end of the outpatient treatment. After three months (TIII) and nine months (TIV) of home treatment, a modified version of this questionnaire was administered by an interviewer to determine the patient's evaluation of the effect of home treatment in terms of changes in lost work and leisure time, dependence on others, and curtailment of activities.

At the beginning of the study, the patient's degree of disability was documented by measuring the range of motion for shoulders, elbows, hips, and knees. ${ }^{13}$ These measurements were repeated on three subsequent occasions. Data on the patient's treatment experience before the study period were obtained from a review of clinical records.

Extended unstructured interviews were also conducted with London patients and selected members of their families; these provided more specific information on problems of adaptation and changes in attitude related to home treatment. In addition, specially designed records documenting the factors associated with each bleed and treatment were completed by each patient and posted to the clinic. Data from these diary sheets were used to record information on cause and site of bleeds, delay before treatment, and recovery time.

- Because of the problems of standardising some details in the treatmen protocol, clinical comparisons were made within each centre population rather than between centres.
The limitations of observing a single group of patients before and after starting home treatment were recognised. However, because commitments had been made in advance to many patients to initiate home treatment as it became available, it was not possible to conduct a randomised or case-matched controlled trial of home and centre-based treatment. In comparing the initial treatment in outpatient centres with subsequent periods of home treatment, only limited control could be imposed for variations in the number and severity of a patient's bleeding episodes during each three-month monitoring period.

TREATMENT METHOD AND SELECTION OF PARTICIPANTS

Patients were selected for the home treatment study on the basis of several criteria: severity, frequency of bleeds, and competence in administering treatment. After screening patients to determine whether they had adequate superficial veins, staff members assessed the personal and familial resources considered necessary for safe home treatment. These included the individual's ability to monitor his own condition and keep accurate clinical records, and the availability of parents, wives, or friends who could give assistance if necessary. Consideration was given to the minimal age at which a patient could undertake self-treatment. Access to a telephone, to facilitate immediate communication with the treatment centre staff in emergencies, was another important requirement for participation in the study. Treatment materials, supplied in bulk to the centres, were made up into sets and freeze-dried concentrates were stored in home refrigerators.

The agreement of a patient's general practitioner was obtained before he entered the study. By the end of the outpatient phase of the study, all patients had received training for home treatment. All patients

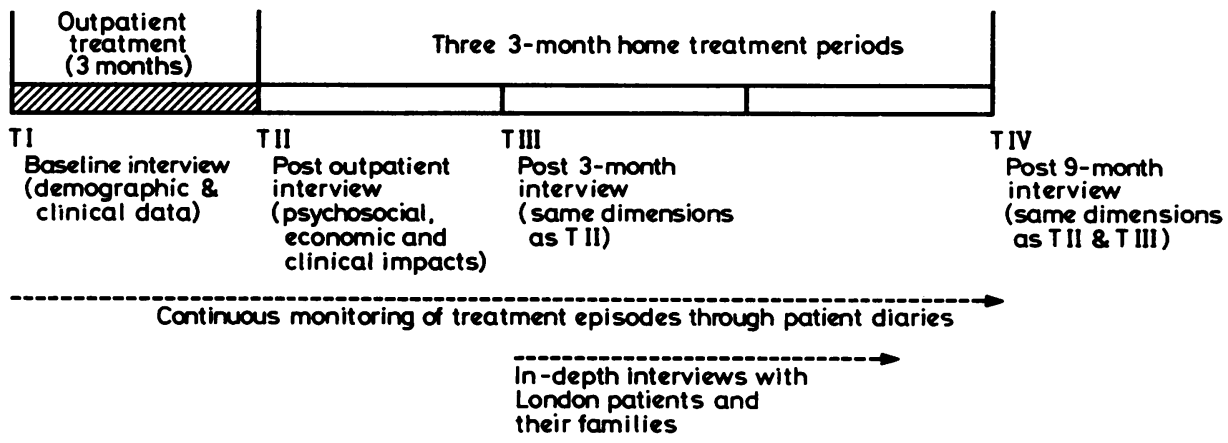

Figure Sequence of data collection. 
were treated with National Health Service freeze-dried Factor VIII concentrate.*

\section{Results}

\section{CLINICAL OUTCOME}

Data on individual bleeding episodes during both phases of the pilot study indicated that delays in receiving treatment and recovery times were reduced during home treatment. The mean values for both Oxford and London patients during the outpatient phase of the project indicated that there was a delay of approximately five hours before treatment. ${ }^{1}$ Delay time was reduced to approximately two hours with home treatment. During home therapy, patients in both groups also recovered from individual bleeds

\footnotetext{
- Participants were instructed to administer one bottle containing ca 250 iu Factor VIII activity as soon as they felt the onset of a bleed. Follow-up doses of one bottle were used by London patients and two bottles by Oxford patients for bleeding episodes which were not relieved 6-12 hours after initial treatment. Major traumatic injuries were treated immediately with two bottles and the treatment centre was notified.
}

more quickly, although reported recovery times were shorter among the London patients. The amount of treatment material required did not materially change from outpatient to home treatment; however, respondents used less analgesic to cope with the pain of developing bleeds. In 1439 individual home treatments, 16 minor reactions were reported by a $\frac{1}{\Phi}$ total of six patients. No severe reaction was reported, $\stackrel{\stackrel{\rho}{F}}{=}$ and a GP was never called. Patients experienced fewer difficulties with the clinical aspects of home $\overrightarrow{\bar{S}}$ treatment than they had anticipated in the baseline interviews.

I M P A C T O F SOCIOD E M G R A P H I

CHARACTERISTICS Clinical outcome measures (summarised in Ingram $e^{\stackrel{\overbrace{}}{\infty}}$ $a l^{1}$ ) did not show any clear age-related differences in $\vec{O}$ treatment rate, delay before treatment, recovery $\vec{\overrightarrow{ }}$ time, or amount of treatment materials used (Table ${ }_{\sigma}^{\omega}$ 1). Age-related differences in the participant's $\frac{\widehat{\sigma}}{\mathrm{C}}$ psychological and social responses to home?

Table 1 Demographic characteristics of the patients

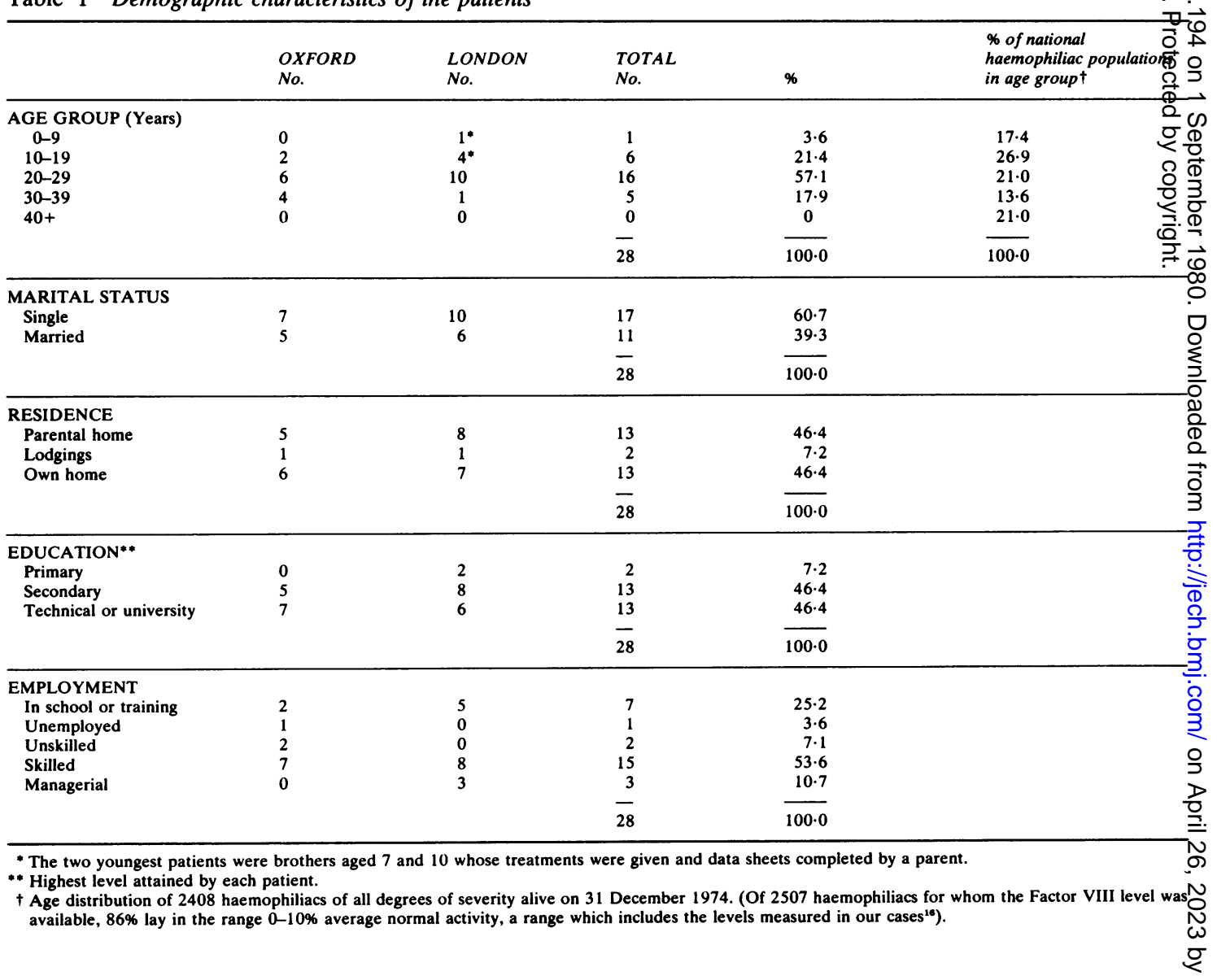


treatment were primarily associated with the characteristics of the family groups involved in treatment, variations in dependency relationships with parents, spouses and friends, and previous experience with less efficacious methods of therapy. The composition of the haemophiliac's family group or household influenced the kind and the range of individuals who provided transport and who were directly involved in assisting with treatment (Table 1).

Fifty-four per cent of the respondents indicated that disability associated with haemophilia or the need to live near treatment centres influenced choice of place of residence and type of housing, or had a major impact on one or more family members' decisions about employment. For patients who no longer lived in their parental homes, choice of location and type of housing continued to be influenced by the treatment priorities. In the unstructured interviews, however, three of the London patients cited specific examples of the effect of available home treatment in removing limitations on place of residence and deçisions about employment.

The duration of the home treatment study obviously did not allow prospective evaluation of the impact of home treatment on educational opportunity. However, the educational background of the participants might have been expected to influence their adaptation and response to home treatment. Patients were grouped into three educational categories (Table 1). Clinical responses to home treatment after three and nine months did not systematically vary in relationship to the patient's level of education. However, among the seven patients who were still enrolled in school or occupational courses, there was a reduction in time lost through treatment of and recovery from bleeds.

It has been suggested that occupational status may affect a haemophiliac's initial capacity to adjust to his disability and to the specific limitations of home treatment. To facilitate rough comparisons, the participants were grouped into five general occupational categories (Table 1). Respondents in most categories indicated that they continued to treat the majority of bleeds at home, despite the availability of treatment in their places of work. Patients whose employers or fellow workers were not aware of their condition, or who worked in areas where it was difficult to initiate treatment, experienced more problems with self-therapy.

\footnotetext{
ADVANTAGES AND DISADVANTAGES OF HOME TREATMENT

The patients' evaluations of the advantages and disadvantages were recorded before and after home treatment. Their responses provided a number of
}

insights into the social, psychological, and organisational factors which influence the perceived efficacy of home treatment. In the interviews, the respondents were asked to rate the level of importance of five major advantages of home treatment on a three-point scale.

The two advantages rated as most important were the reductions in time spent travelling for treatment and in time lost from work. The patients' evaluation of the importance of the reduction in travel time increased after three and nine months of home treatment. Their perception of the importance of this advantage is supported by the analysis of individual treatments. This shows marked reductions not only in travel costs but also in the total costs of managing individual bleeding episodes. ${ }^{*}$

The benefit of home treatment ranked third was reduction in time lost from social activities to treat bleeds. Advantages associated with reduced dependence on the treatment centre and on members of the family were rated as fourth and fifth (that is, less important than reduction of direct and indirect time and monetary costs).

The TII, TIII, and TIV questionnaires included four structured questions asking the participants to rate the importance of possible disadvantages of home treatment associated with the preparation of materials, administering treatment, increased family involvement, and lack of medical supervision. The potential disadvantages which were perceived as being 'somewhat important' before home treatment began were the preparation and administration of treatment materials; one-quarter of the patients expressed concern about preparing and administering materials without clinical supervision and about obtaining assistance from members of their families in performing venepunctures. During home treatment, three patients reported difficulties in transporting and storing materials. However, all patients rated these disadvantages as unimportant after three and nine months of home treatment.

In response to open-ended questions after three and nine months of home treatment, respondents were able to describe specific changes in their own patterns of symptomatology and dependence. After three months, over half of the patients were able to cite specific examples of their increased capability to respond to the symptoms of an oncoming bleed more rapidly. Four respondents mentioned that whereas previously they had frequently left developing bleeds untreated because of the problems of obtaining

\footnotetext{
- The mean cost of work and leisure time lost per bleeding episode for Oxford patients dropped from $£ 72.50$ during centre-based outpatient treatment to $£ 48$ after three months and $£ 38$ after nine months of home treatment. Among the St. Thomas's patients the cost per bleed dropped from $£ 34.50$ during outpatient treatment to £26 after three months and $£ 22$ after nine months of home treatment.
} 
immediate treatment, with home therapy available they were in a better position to begin treatment when symptoms first appeared.

Another common theme was the impact of home treatment on the patient's capacity to plan his activities and minimise demands on friends and family members. More than half of the participants cited the effect of home treatment in minimising social opportunity costs, direct financial costs of transport, and lost work time. Among the most immediate effects were changes in the patients' capacity to organise their time and plan social activities in advance. Of the 28 patients, $15(54 \%)$ referred to these advantages before home treatment and $24(86 \%)$ after three months' treatment. Increased mobility and greater confidence and willingness to become involved in social activities were also mentioned.

Patients were also asked to describe changes in their functional abilities and willingness to take risks. Descriptions of high-risk activities and personal adaptation to these risks varied greatly from one individual to another, as did the activities avoided because of the level of associated risk. The majority of respondents indicated that it was difficult for them to identify a set of activities which constituted 'normal' functions because their experience had restricted the range of activities perceived to be 'possible'. However, specific questions on the use of public transport and on holiday travel were included to investigate changes in these areas of functional ability. Only two of the nine patients who had described themselves as 'unwilling' to use public transport during outpatient treatment altered their evaluation of the associated risk during home treatment. However, the patients' willingness to take risks associated with travelling and going on holiday seems to have been more directly influenced by the home treatment experience. The proportion who indicated that they were willing to travel away from the vicinity of their centre rose from $10(36 \%)$ during outpatient treatment to $23(82 \%)$ after three months and to $100 \%$ after nine months of home treatment. Changes in attitudes to travel may reflect the effect of available home therapy in minimising the logistical problems, rather than any fundamental change in attitudes towards risk-taking.

\section{FAMILY SUPPORT AND DEPENDENCY RELATIONSHIPS}

A central issue in assessing the impact of home treatment is the effect of involving spouses, parents, or other members of the household in therapy. Recent literature examining the role played by the family in home therapeutic regimens has focused on the effect of giving prime control of treatment to the patient or another member of the family, the pressures on those who are helping with the treatment, and the impact of the treatment on dependency patterns within the family. ${ }^{14}$ The way in which members of the family or household are involved in treatment has been identified as aT significant variable in home therapy; giving the helper the dominant role in treatment may give $\underset{\text { \$. }}{\text {. }}$ disproportionate amount of initiative, responsibility $\overrightarrow{\vec{z}}$ and control to a family member or friend and reinforce the patient's dependent status. ${ }^{15}$

To avoid creating a situation of that kind, each participant kept control of his own treatment? regimen. Members of the family were either broughi in for parallel training during the clinical treatmentes phase or trained by the haemophiliac himself. Duringthe training phase of outpatient treatment, the staff of the haemophilia centre assessed the potentiat $\vec{\omega}$ contribution of the network of family members and? friends. Family support and the presence of memberso willing to become involved in treatment w\&e $e_{\omega}^{\supset}$ frequently mentioned by clinical staff in explaini variations in the patients' adaptations to home treatment. Another crucial advantage of horge 0 treatment was that it increased the patient's cont $\$$ lo in performing basic self-care functions. He was betefer $\mathrm{r}_{-}^{?}$ able to plan his requirements for assistance fromes family and friends, and crises emphasising dependency and inability to exercise contro occurred less frequently.

Family members were involved in two areas home treatment: setting up and preparing the freeze- $\overrightarrow{0}$ dried material, and doing the venepuncture. After the ${ }^{\infty}$ three-month centre-based training phase of the project, $16(57 \%)$ of the participants had one oro more individuals in their families or households, or had access to a school nurse or occupational health nurse, able to assist with the preparation of treatment $\frac{\alpha}{\not}$ materials. The level of family involvement in 0 performing the venepuncture was somewhat lower. $\overrightarrow{0}$ Only six participants involved members of their 3 families in performing venepunctures at the beginning of the initial period of home treatment; in? addition, one patient's wife became involved during the first three months. Only one patient reported that $\stackrel{\sim}{?}$ he had had difficulty in obtaining the assistance required from members of the family. In the open- 3 . ended interviews, the majority of members of the London patients' families indicated that specific $₹$ information about the treatment regimen and moreo general information about the implications of haemophilia for disability had increased their abilityo to support the patient and assist with therapy.

A primary objective of the comparison of outpatient and home therapy was to measure the N dependency patterns under each mode of treatment. N 
Changes in dependency can be measured in terms of levels of physical reliance on others for assistance and psychosocial assessment of perceived reliance on others. In developing measures of outcome, it proved difficult to utilise a standardised inventory of primary functions, such as Katz's Activities of Daily Living ${ }^{11}$ among a sample with wide variations in levels of disability and long histories of physical limitation.

However, open-ended questions were included to allow participants to elaborate on the specific tasks for which they most frequently needed help. Although there was great variation among individuals, functions such as dressing, eating, and washing were the activities for which assistance was most frequently required. More complex functions such as shopping, gardening, and repair work were also mentioned. Wives and parents most frequently helped with these.

Although $82 \%$ of the respondents felt that home treatment would minimise the period of disability associated with a bleed, most foresaw situations in which they would continue to be dependent on members of their household for assistance with activities of daily living or for help with treatment. Seven patients emphasised in the open-ended interviews that changes in patterns of dependence had to be evaluated in the light of experience of longer-term effects.

The limited duration of the home treatment and the reluctance of participants to respond to projective psychological tests influenced the decision of the study team to use a simple summary measure of patients' perceptions of their level of dependence or reliance. After the detailed elaboration of situations in which they required assistance, respondents were asked to describe their levels of dependence in terms of three summary categories. In this way, self-rated dependence was related to the detailed description of the respondent's need for assistance from members of his family or wider social network. Summary ratings of perceived dependence on others changed noticeably with home treatment experience (Table 2 ). The proportion of participants who characterised themselves as 'not reliant' rose from $39 \%$ at TII, to $61 \%$ at TIII and to $81 \%$ at TIV. Five patients characterised themselves as 'very reliant' before home treatment (TII) compared with only two at TIII and only one at TIV.

\section{Conclusions}

The benefits of home therapy to patients may be estimated by measures of clinical outcome, such as treatment delay or recovery time, and by measures of opportunity costs, such as time lost from school or work. The London-Oxford study of the differences
Table 2 Perceived dependence or reliance on others $(n=26)$

\begin{tabular}{lllll}
\hline & $\begin{array}{l}\text { TII } \\
\text { (Post-treatment } \\
\text { centre phase) }\end{array}$ & $\begin{array}{l}\text { TIII } \\
\text { (After } 3 \text { months of } \\
\text { home treatment) }\end{array}$ & $\begin{array}{l}\text { TIV } \\
\text { (After 9 months of } \\
\text { home treatment) }\end{array}$ \\
\hline Very reliant & (5) $19 \%$ & (2) $8 \%$ & (1) $4 \%$ \\
\hline Somewhat reliant (11) $42 \%$ & (8) $31 \%$ & (4) $15 \%$ \\
\hline Not reliant & (10) $39 \%$ & (16) $61 \%$ & (21) $81 \%$
\end{tabular}

The two youngest participants, whose mother was involved in the interview, were not asked to rate their overall level of dependency.

between outpatient and home treatment would appear to substantiate claims that such benefits do exist. However, the observation period was too short to obtain data comparable to those available for hospital-based treatment.

Like the other measures of outcome, changes in the patterns of dependence exhibited by the participants can be interpreted only within the context of the limited duration of the study. Nevertheless, the degree of change observed with only nine months of home therapy does underline its significance to the patient. The short-term effect is seen in the immediate increase in the level of control a patient exercises over treatment and the decrease in the use of the treatment centre; the patient's ability to travel in areas without easy access to a centre is another indication of a decrease in dependence. His capacity to plan his activities, treat himself, and perform some self-care functions during bleeding episodes effectively minimises demands on friends or members of his family.

The evaluation of the effects of home treatment made by the haemophiliacs themselves emphasised that these outcomes were immediately experienced; more profound changes will require longer-term documentation. However, the London-Oxford study suggests that home therapy will influence the disability patterns of the present generation of haemophiliacs, particularly through changing their perceptions of themselves and their capacity to deal with the problems of their disease. As well as immediate, practical benefits like increased mobility and less time lost from work and school, the participants' greater confidence and willingness to become involved in social activities, and the decrease in their perceived reliance on others, indicate the direction of future changes in patterns of dependency, career development opportunities, and familial relationships among haemophiliacs.

I thank the following members of the multidisciplinary team who carried out the overall study: G. I. C. Ingram, S. R. Dykes, A. L. Creese, P. 
Mellor, and A. V. Swan (St. Thomas's Hospital Medical School, London); and C. R. Rizza, R. J. D. Spooner, and R. Biggs (Churchill Hospital, Oxford). We are grateful to the 28 patients for their contribution to the pilot study. I also thank J. Peterson, J. Kearns, Professor W. W. Holland, Professor D. G. Fish, and Professor E. Shapiro for editorial comments and Ms. P. Roberts and Mrs. C. Guenette for help in preparing the manuscript. The project was supported by a small grant from the Welsh Office.

Reprints from Professor J. Kaufert, Department of Social and Preventive Medicine, University of Manitoba, 750 Bannatyne Avenue, Winnipeg, Manitoba, Canada R3E OW3.

\section{References}

' Ingram GI, Dykes SR, Creese AL et al. Home treatment in haemophilia: clinical, social and economic advantages. Clin Lab Haematol 1979; i: 13-27.

${ }^{2}$ Levine PH. Efficacy of self-therapy in hemophilia. $N$ Engl J Med 1974; 291: 1381-4.

${ }^{3}$ Evensen SA, Thaule R, Groan K. Self-therapy for haemophilia in Norway. Acta Med Scand 1979; 205: 395-9.

${ }^{4}$ Rizza CR, Spooner RJD. Home treatment of haemophilia and Christmas disease: five years' experience. $\mathrm{Br} J$ Haematol 1977; 37: 53.
${ }^{5}$ Anonymous. Haemophilia and home therapy. Lancet 1979; ii: 77-8.

${ }^{6}$ Haemophilia Society. Special reports: on Employment and Unemployment; Social Welfare; Education. Survey of Adult Haemophiliacs 1967-68. London: Haemophilia Society, 1968, 1977.

${ }^{7}$ Haemophilia Society. Report on a Survey of Adult Haemophiliacs. (Mimeo). London: Haemophilia Society, 1978.

${ }^{8} \mathrm{Katz}$ S. Some psychosocial problems in hemophilia. Soc Casework 1959; 40: 321.

${ }^{9} \mathrm{Katz}$ S. Social adaptation in chronic illness: a study of $\stackrel{\vec{S}}{\rightarrow}$ hemophilia. Am J Public Health 1964; 53: 1666.

${ }^{10}$ Salk L, Hilgarthner M, Granick B. The psychosocial impact of haemophilia on the patient and his family. Soc Sci Med 1972; 6; 491.

${ }^{11} \mathrm{Katz}$ S. Activities of Daily Living in Assessment.

${ }^{12}$ Forbes C, Markova I. Progress Report 1974-1975. ल) Self-perception of risk-taking behaviour of $\overrightarrow{0}$ Haemophiliacs. Report of the Department of Medicine, Glasgow Infirmary, Glasgow, 1975.

${ }^{13}$ American Academy of Orthopaedic Associations. Joint Motion: Method of Measuring and Recording. New York: American Academy of Orthopaedic Associations, 1965.

${ }^{14} \mathrm{Litman}$ TJ. The family as a basic unit in health and medica care: a social behavioural overview. Soc Sci Med 197\% $\omega$

8: 495.
${ }^{15}$ Gallagher EB. Home dialysis and sociomedical poliç. Paper presented at the British Sociological Associatiog conference, Manchester, England, April 1976.

${ }^{16}$ Biggs R. Haemophilia treatment in the United Kingdof from 1969-1974. Br J Haematol 1977; 35: 487. 